\title{
Printed Circuit Board Defect Detection Using Mathematical Expression in Image Processing
}

\author{
R. VijayaGeetha, M. VijayaBharathi \\ Department of Electronics and Communication Engineering \\ Dr. T. Thimmaiah Institute of Technology, Kolar Gold Field, Karnataka \\ vijayageetha@drttit.edu.in
}

\begin{abstract}
. in electronic circuit board, numerous methods have been developed to detect the faults presents on printed circuit board (PCB). It is important to identify the fault in order to investigate and recognize the root source of the defects. This method aims to identify and classify the faults presents on bare layered single PCB using easy mathematical expression. Proposed method have utilized by simple arithmetic and logical equations of NOT, SUBTRACT and XOR operation in image processing tool. Resultant image are classified by binary thresholding. Test image and printed PCB images have used for experiments and identified seven various defects on them. In industrial purpose, testing the manufacturing defects on huge number of PCBs using Image processing leads less consume time and effective way of detecting fault in metal prints.
\end{abstract}

Keywords: Arithmetic and logical expression, image processing and detection rate.

\section{Introduction}

As PCB is most important platform for the assembling of the electronic and mechanical components for final product outcome, on a composite, conductive and non-conductive layer sandwich. In general, the conductive layer is copper although aluminum, chromium and other metals are used, usually the non-conductive layer is a Epoxy and glass fibre-composite. With well developed circuitry with high productivity, boost complexity and circuit board performance, quality of the PCB can impact not only product efficiency but also influence on public protection. So it is very important, the components are placed correctly and exact copper routing is achieved for proper functioning of the system. Ambient, Temperature, Equipment, and defective activity will cause fault presence. Because of unavoidable defects occurrence in the manufacture of PCBs such as short circuit, Circuit breach, burr, fault, hole and other defects.

If any faults are found in the advanced stages of PCB production like etching of the extra copper, we have to discard the PCB [1]. Hence to avoid such faults, we adopt image processing technique to detect and correct the errors before the PCB goes for assembling of the components. The inspection of PCBs has two main processes: identification of defects and classification of defects. There are currently several algorithms 
for detecting PCB defects, using touch or non-contact methods [3]. The background techniques for PCB defect detection designed by primitive patterns of the PCB image and clustering methods have used. The clustering methods have four important segments of patterns using mathematical frame of morphological operation and windowing method. Then they have classified nearly 12 defects out of the 14 types of bare single PCB layer using image segmentation by artificial neural network. The performance of software design tool using MATLAB designed by image subtraction methods using XOR, NOT and IMFILL operation and found nearly 14 defects grouped of five classes [1]. In this paper [2], [3], used hybrid methods to produce an improvement and found defects of 14 grouped into seven classes. This method is combined the segmentation algorithm with image processing techniques. This combined technique produced five classified images for comparison of reference and test image.

Table 1 Types of defects in bare PCB layer

\begin{tabular}{|cl|cl|}
\hline No. & Defect Type & No. & Defect Type \\
\hline 1 & Break-out & 8 & Sunken \\
2 & Pin-hole & 9 & Hump \\
3 & Open circuit & 10 & Wrong size hole \\
4 & Under-etch portion & 11 & Missing conductor \\
5 & Mouse-bite area & 12 & Spurious Copper \\
6 & Spur & 13 & Missing hole \\
7 & Short & 14 & Excessive short \\
8 & Sunken & 15 & Over-etch portion \\
\hline
\end{tabular}

\section{Methodology}

Defect detection is the important concept of electronic circuit board. Defects are mainly appears as extra connectivity print in metal layers or focal connection may be missing in the printing layer of the board. There are two important errors affect the circuit board; surface defects and design defects. The production unit of circuitry board is based on chemical and mechanical events that may scratch the anticipated design. Frequently different types of defects may affect the PCB are open circuit, lost conductor, Shorting, incorrect size hole, lost hole, breakout and pin-hole [4][9].Image processing plays an essential role for defect detection in ease way and accurate manner. The simple arithmetical and logical expressions are used to predict the defects in production units. The arithmetic equation of image subtraction is used between main reference PCB and defected PCB images. [8] Subtraction procedure produce negative image defects as well as positive image defects to monitor the imperfection caused in missing prints and overlay of faults [5]. Negative defect image is subtraction of reference image to the test image; positive defect image is subtraction of test image to the reference image. The logical expressions of NOT operation gives complement structure to represents major faults of 
lost conductor, under-etch and over-etch connection [6][11]. Exclusive-OR operation functions produce the result image if only precisely one of the differences is present [7][10]. The mathematical expression are given below as,

Logical expression

NOT: Output $=\sim$ Test image

XOR: $\quad$ Output $=$ Referenceimage $\oplus$ Testimage

Arithmetic expression

Negative defect image: Subtract: $\quad$ Output $=$ Input $1-$ Input2

Positive defect image: Subtract: $\quad$ Output $=$ Input $2-$ Input 1
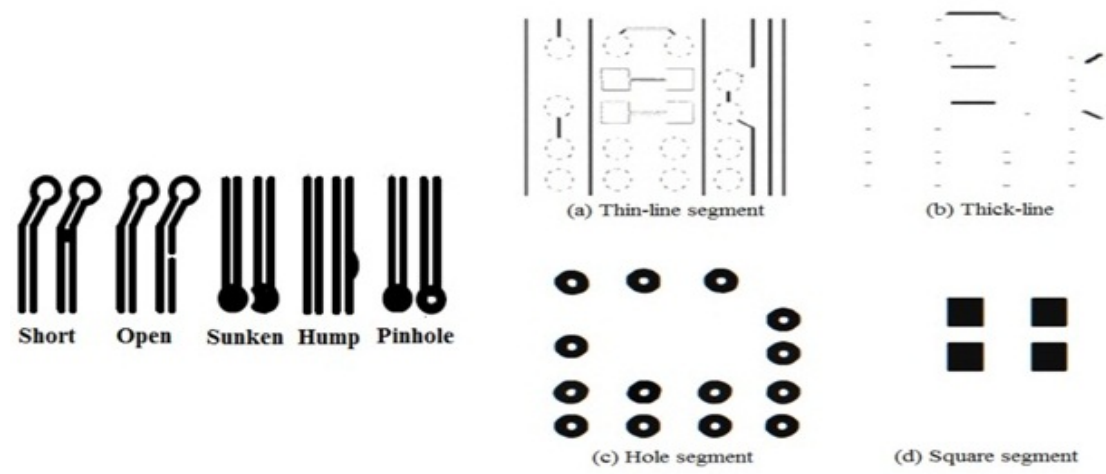

Fig. (1) Possible defects of PCB and defect features

\section{Thresholding Method}

Threshold is most important segmentation method in image processing. It allows gray scale image into binary image. After determine threshold value of each pixel brightness value will replace by according to threshold value. The proposed threshold algorithm that estimates and locates an optimum threshold $\hat{r}$ for unknown probability that can be used to classify each pixel into two possible classes of change and no change with condition given below:

$$
\begin{aligned}
& \text { If }_{i j} \leq \hat{r} \\
\text { Thenr }_{i j} & \rightarrow \text { NoChangeclass } \\
\text { Elser }_{i j} & \rightarrow \text { Changeclass }
\end{aligned}
$$

The kittler Illingworth algorithm is popular method for identify the change region, usually carried out by applying threshold method to find histogram of the difference image. 


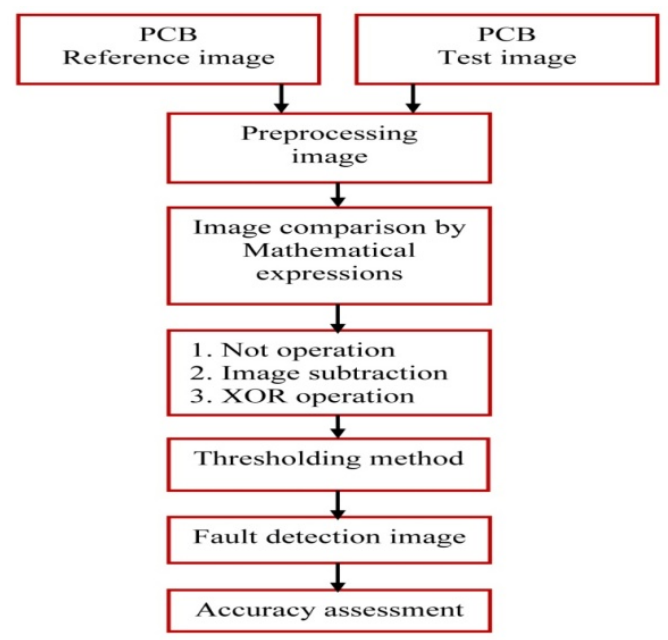

Fig. (3) PCBs Defect detection flow chart

To select the optimum threshold for defect detection that minimizes the criterion function give in (3)

$$
J\left(r_{T}\right)=-\sum_{i=1,2}\left[P_{i}\left(r_{T}\right) \cdot P_{i}\left(r_{T}\right)+\sum_{r \in i} h(r) \ln P_{i}\left(\frac{r}{r_{T}}\right)\right](3)
$$

Where refers to the defect and non-defected classes respectively. These criterion functions appeared in prior probability function and conditional probability function is used to estimate specific threshold value.

\section{$4 \quad$ Result and Discussion}

The defect detection is tested by mathematical expression using primary operation of NOT operation, image subtraction and XOR operation. Figure (4) represents the output results compared by subtraction operation and described as Fig. 4(a) test RGB image, this image has preprocessed by RGB to binary conversion and also filtered by median filter. The reference image and test image has to compare by subtraction techniques, those images are aligned with equal pixel matching. Figure 4(b), (c) are reference and test images. Figure (d), (e) are the complementary images to highlights the line features and Missing conductor faults. Figure 4(f), (g) represents image subtraction also gives defects in negative image and positive image, which gives results of image with defects of thin line, breakout, pin-hole, missing conductor and wrong size hole have identified. Figure 4(h) is absolute image subtraction describes the subtraction with only positive pixel values, this result gives non-negative representation of the image. Figure 4(i) represents the exclusive OR operation to compare the reference image and the test image which gives changes presents in both the classes. Figure 5 is the bare single PCB board compared the reference image and the test PCB image. This method is analyzed by XOR operator and observed minute defect also have predicted. Figure 6 represents the 
known defects are classified and are marked in the each specific feature. The table 1 represents the quality measure of the defect detection on the test and the PCB image. The comparative result shows that XOR method based subtraction gives effective fault appraisal result compare than other existing methods.

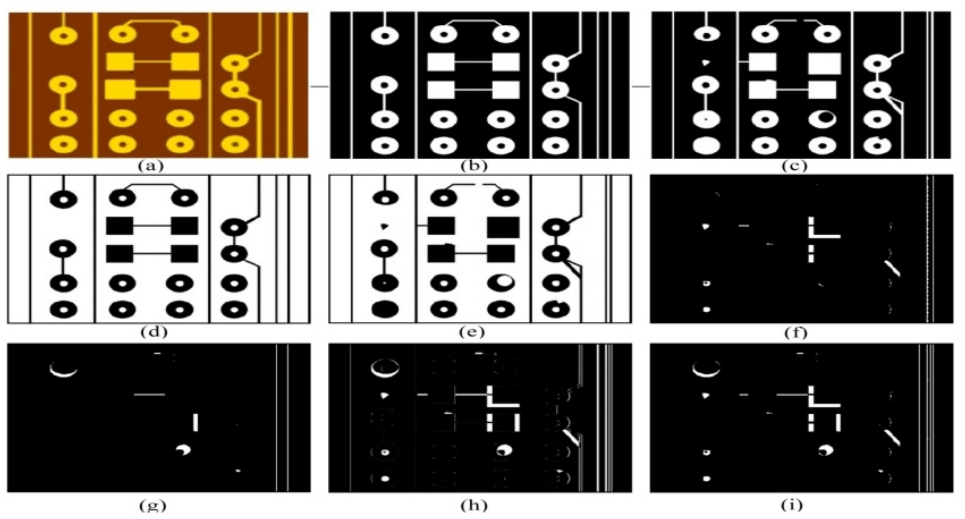

Fig. (4) Experimented results on test image (a) RGB image of PCB, (b) Reference image, (c) Test image,

(d) NOT operation on reference image, (e) NOT operation on test image, (f) Negative defect image by subtraction (g) Positive defect image by subtraction, (h) Absolute difference, (i) XOR operation with thersholding

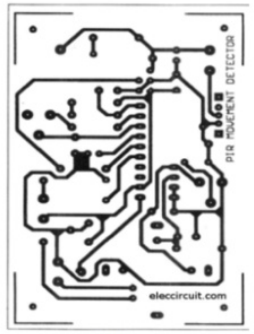

(a)

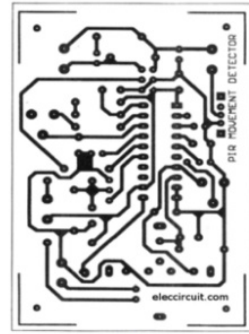

(b)

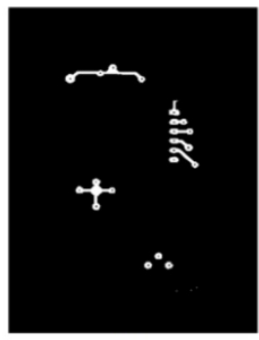

Fig. (5) Experimented results on PCB image (a) reference image, (b) single PCB image (c) XOR with thresholding

Table 2. Quality measure of defect detection on the reference image and the test image

\begin{tabular}{|c|l|c|c|c|c|}
\hline Dataset & Method & DDR & AR & FAR & ER \\
\hline \multirow{3}{*}{ Test image } & Image Subtraction & 0.4578 & 0.6248 & 0.7586 & 0.4758 \\
\cline { 2 - 6 } & Absolute difference & 0.7458 & 0.8745 & 0.5548 & 0.1854 \\
\cline { 2 - 6 } & XOR Operation & 0.8745 & 0.9746 & 0.4558 & 0.0476 \\
\hline \multirow{3}{*}{ PCB image } & Image Subtraction & 0.5657 & 0.6778 & 0.7756 & 0.5758 \\
\cline { 2 - 6 } & Absolute difference & 0.6488 & 0.8854 & 0.5758 & 0.9752 \\
\cline { 2 - 6 } & XOR Operation & 0.8975 & 0.9655 & 0.4358 & 0.0376 \\
\hline
\end{tabular}




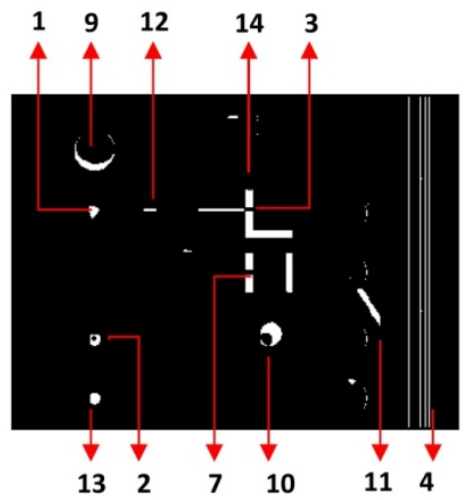

Defect detected

\begin{tabular}{|c|l|}
\hline No. & \multicolumn{1}{|c|}{ Defect Type } \\
\hline 1 & Break-out \\
\hline 2 & Pin-hole \\
\hline 3 & Open circuit \\
\hline 4 & Conductor too close \\
\hline 7 & Short \\
\hline 9 & Hump \\
\hline 10 & Wrong size hole \\
\hline 11 & Missing conductor \\
\hline 12 & Spurious Copper \\
\hline 13 & Missing hole \\
\hline 14 & Excessive short \\
\hline
\end{tabular}

Fig. (6) Classified Defected portion 


\section{Conclusion}

The experimental result shows that the arithmetical and logical expression can find the regular defects on the printed circuit board. The combined method of XOR operation with Kittler-Illingworth segmentation method provides better result. XOR operation could able to identify the defects presents in both of the images, hence missing or overlay problem would not present. Kittler Illingworth algorithm gives effective segmentation of defect and non-defect positions. By this method 13 types of the defects can detect out of 15 defects. By this experiment results, the performance analysis are comparing and provided better defect detection rate of $0.8745,0.8975$ for test image and PCB image respectively. Classified defected portions are listed with defect types. This method is easy to implement for testing and identifying faults in PCB manufacturing industries.

\section{References}

1. M. Moganti, F. Ercal, "Automatic PCB inspection Algorithms: A Survey," Computer Vision and Image Understanding, vol. 63, no. 2, pp. 287-313, 1996.

2. T. Taniguchi, D. Kacprzak, S. Yamada, M. Iwahara, and T. Miyogashi, 'Defect Detection on Printed Circuit Board by using Eddy-Current Technique and Image Processing', 101 Press, 2000

3. Wen-Yen Wu, Mao-JiunJ.Wang and Chih-Ming Liu, 'Automated Inspection of Printed Circuit Board Through Machine Vision', Computers in Industry, 28 (1996) pp.I03-III.

4. Z. Ibrahim, S.A.R AI-attas and Z. Aspar, 'Analysis of the WaveletBased Image Difference Algorithm for PCB Inspection', in proceedings of SICE, Osaka, Japan, 2002

5. Z.Ibrahim, S.A.R Al-attas and Z.Aspar, 'Coarse Resolution Defect Localization Algorithm for an Automated Visual PCB Inspection', Journal Teknologi, 37(D), Dis. 2002, 79-92.

6. S. T. Ahmed, "A study on multi objective optimal clustering techniques for medical datasets," 2017 International Conference on Intelligent Computing and Control Systems (ICICCS), Madurai, 2017, pp. 174-177, doi: 10.1109/ICCONS.2017.8250704.

7. K. D. Singh and S. T. Ahmed, "Systematic Linear Word String Recognition and Evaluation Technique," 2020 International Conference on Communication and Signal Processing (ICCSP), Chennai, India, 2020, pp. 0545-0548, doi: 10.1109/ICCSP48568.2020.9182044

8. S. T. Ahmed, H. K. Priyanka, S. Attar and A. Patted, "Cataract density ratio analysis under color image processing approach," 2017 International Conference on Intelligent Computing and Control Systems (ICICCS), Madurai, 2017, pp. 178-180, doi: 10.1109/ICCONS.2017.8250705.

9. Gunashree, M., Ahmed, S. T., Sindhuja, M., Bhumika, P., Anusha, B., \&Ishwarya, B. (2020). A New Approach of Multilevel Unsupervised Clustering for Detecting Replication Level in Large Image Set. Procedia Computer Science, 171, 1624-1633. https://doi.org/10.1016/j.procs.2020.04.174

10. J. Dafni Rose, K. Vijayakumar and S. Sakthivel, "Students' performance analysis system using cumulative predictor algorithm", Int. J. Reasoning-based Intelligent Systems, Vol. 11 , No. 2, 2019

11. K. Vijayakumar, Chokkalingam Arun, "Integrated cloud-based risk assessment model for continuous integration", Int. J. Reasoning-based Intelligent Systems", Vol. 10, Nos. 3/4, 2018. 